\title{
REVIEW
}

\section{Urinary biomarkers for prostate cancer: a review}

\author{
Daphne Hessels ${ }^{1}$ and Jack A Schalken ${ }^{2}$
}

\begin{abstract}
Although the routine use of serum prostate-specific antigen (PSA) testing has undoubtedly increased prostate cancer (PCa) detection, one of its main drawbacks is its lack of specificity. As a consequence, many men undergo unnecessary biopsies or treatments for indolent tumours. PCa-specific markers are needed for the early detection of the disease and the prediction of aggressiveness of a prostate tumour. Since PCa is a heterogeneous disease, a panel of tumour markers is fundamental for a more precise diagnosis. Several biomarkers are promising due to their specificity for the disease in tissue. However, tissue is unsuitable as a possible screening tool. Since urine can be easily obtained in a non-invasive manner, it is a promising substrate for biomarker testing. This article reviews the biomarkers for the non-invasive testing of $\mathrm{PCa}$ in urine.
\end{abstract}

Asian Journal of Andrology (2013) 15, 333-339; doi:10.1038/aja.2013.6; published online 25 March 2013

Keywords: biomarkers; diagnosis; prostate cancer (PCa); urinary biomarkers; urine

\section{INTRODUCTION}

Annually, 241740 men in the United States and 338700 men in Europe are newly diagnosed with prostate cancer $(\mathrm{PCa})$ and around 28170 US and 70800 European men die from this disease. ${ }^{1,2}$ Early detection of PCa relies on serum prostate-specific antigen (PSA) testing or digital rectal examination (DRE). Since its first clinical application, serum PSA has been a valuable tool in the detection, staging and monitoring of this disease. Although the routine use of serum PSA testing has undoubtedly increased PCa detection, one of its main drawbacks has been its lack of specificity resulting in a high negative biopsy rate. ${ }^{3}$ The early detection of many indolent PCas has resulted in treatment of tumours that would not have become life-threatening to a patient.

Serum PSA has a low specificity because it is not a PCa-specific event; elevated levels can also be detected in men with benign prostatic hyperplasia (BPH) and prostatitis. Methods to enhance PSA specificity have assisted clinicians in deciding which patients should undergo biopsy, but have not necessarily improved diagnostic accuracy or facilitated optimal therapeutic decision-making. More accurate tests that can stratify patients according to their risk of developing PCa, identify men who require repeat prostate biopsy and stratify men at risk for aggressive disease are needed.

Many biomarkers have been identified and some of them are promising due to their specificity for the disease in tissue. However, tissue is unsuitable as substrate for biomarker testing because of its invasiveness and expensiveness. Therefore, testing of disease-related biomarkers in body fluids that can be obtained in a non-invasive manner seems a good alternative as possible screening tool. Because of the ease of collection, and the fact that prostate cells are directly released into the urethra through prostatic ducts after DRE, urine has become the future for non-invasive biomarker testing. This review focusses on the available data concerning the applicability of promising DNA or
RNA-based urinary biomarkers in the early detection and prediction of aggressiveness of PCa (summarized in Table 1).

\section{EPIGENETIC MODIFICATIONS}

Alterations in DNA, without changing the order of bases in the sequence, often lead to changes in gene expression. These epigenetic modifications include changes such as DNA methylation and histone acetylation/deacetylation. Many gene promoters contain GC-rich regions also known as $\mathrm{CpG}$ islands. Hypermethylation of $\mathrm{CpG}$ islands results in decreased transcription of the gene into mRNA. Recently, it was suggested that the DNA methylation status may be influenced in early life by environmental exposures, such as nutritional factors or stress, and that this leads to an increased risk of cancer in adults. ${ }^{4}$ Changes in DNA methylation patterns were observed in many human tumours. ${ }^{5}$ A technique known as methylation-specific PCR (MSP) is used for the detection of promoter hypermethylation. In contrast to microsatellite or loss of heterozygosity analysis, this technique requires a tumour-to-normal ratio of only $0.1 \%-0.001 \%$. This means that using this technique, hypermethylated alleles from tumour DNA can be detected in the presence of $10^{4}-10^{5}$ excess amounts of normal alleles. ${ }^{6}$ Therefore, DNA methylation can serve as a useful marker in cancer detection. Recently, there have been many reports on hypermethylated genes in human PCa. Two of these genes are gluthathione $S$-transferase P1 (GSTP1) and Ras-association domain family protein isoform A (RASSF1A).

\section{GSTP1}

The most described epigenetic alteration in PCa is the hypermethylation of the GSTP1 promoter. GSTP1 belongs to the cellular protection system against toxic effects and as such, is involved in the detoxification of many xenobiotics. GSTP1 hypermethylation was reported in approximately $6 \%$ of the proliferative inflammatory atrophy lesions

${ }^{1}$ NovioGendix BV, Nijmegen, 6525 GA, The Netherlands and ${ }^{2}$ Department of Urology, Radboud University Nijmegen Medical Centre, Nijmegen, 6500 HB, The Netherlands Correspondence: Professor JA Schalken (j.schalken@uro.umcn.nl)

Received: 1 December 2012; Revised: 7 January 2013; Accepted: 8 January 2013; Published online: 25 March 2013 
Table 1 Biomarkers and their potential application in the urinary detection of prostate cancer

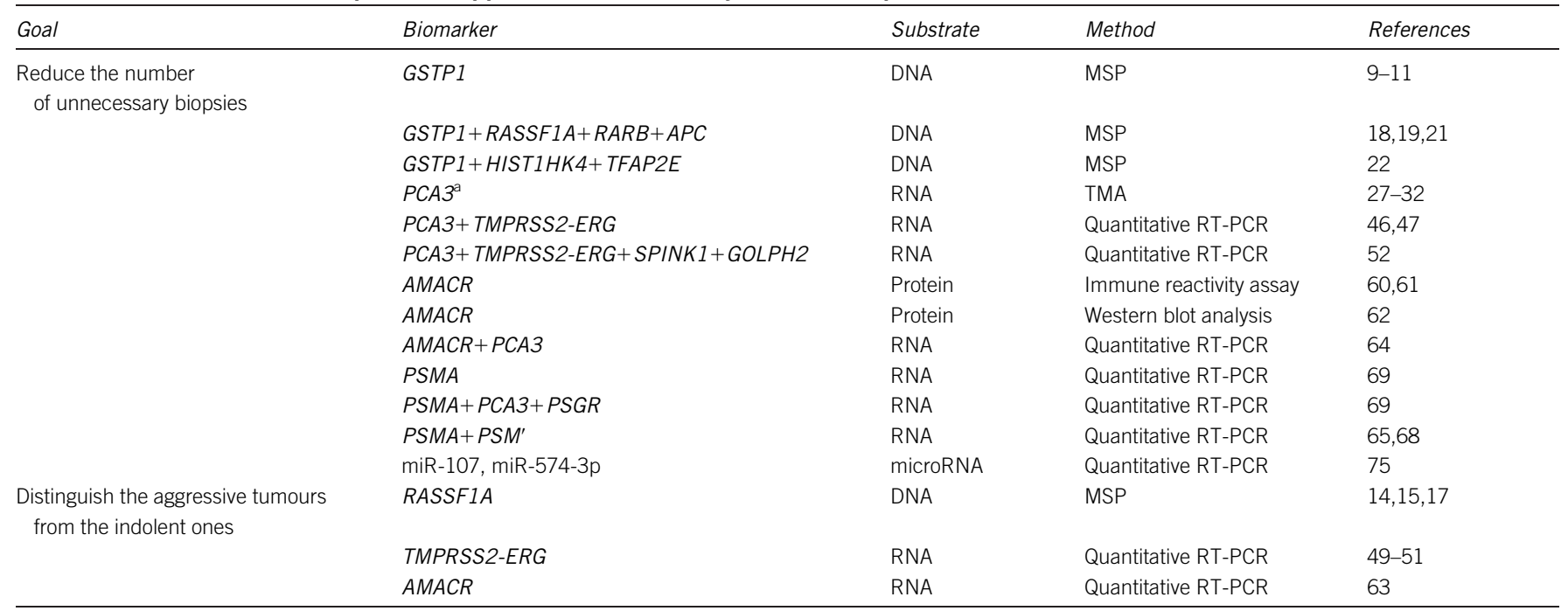

Abbreviations: AMACR, $\alpha$-methylacyl-CoA racemase; APC, adenomatous polyposis coli; GOLPH2, golgi phosphoprotein 2; GSTP1, gluthathione S-transferase P1; MSP, methylation-specific PCR; PSMA, prostate-specific membrane antigen; RARB, retinoic acid receptor $\beta 2$; RASSF1A, Ras-association domain family protein isoform A; RT$P C R$, reverse transcription-PCR; SPINK1, serine peptidase inhibitor Kazal type 1; TMA, transcription-mediated amplification; TMPRSS2, transmembrane-serine protease gene.

${ }^{a}$ FDA approved molecular marker for prostate cancer: Progensa PCA3 test.

and in $70 \%$ of the prostate intraepithelial neoplasia (PIN) lesions. ${ }^{7}$ It was shown that some proliferative inflammatory atrophy lesions merge directly with PIN and early carcinoma lesions, although additional studies are necessary to confirm these findings. Hypermethylation of GSTP1 was detected in more than $90 \%$ of prostate tumours, whereas no hypermethylation was observed in $\mathrm{BPH}$ and normal prostate tissues. ${ }^{8}$

Hypermethylation of the GSTP1 gene was detected in $50 \%$ of ejaculates from PCa patients but not in ejaculates of men with BPH. Due to the fact that ejaculates are not always easily obtained, hypermethylation of GSTP1 was determined in urinary sediments obtained from PCa patients after prostate massage. Cancer could be detected in $77 \%$ of these sediments. ${ }^{9}$ Moreover, hypermethylation of GSTP1 was found in post-prostate massage urinary sediments of $68 \%$ of patients with early confined disease, $78 \%$ of patients with locally advanced disease, $29 \%$ of patients with PIN and $2 \%$ of patients with BPH. These findings resulted in a specificity of $98 \%$ and a sensitivity of $73 \%$. The negative predictive value of this test was $80 \%$, indicating that this assay bears potential to reduce the number of unnecessary biopsies. Recently, these results were confirmed and a higher frequency of GSTP1 methylation was observed in the urine of men with stage $3 v s$. stage 2 disease. ${ }^{10}$ Because hypermethylation of GSTP1 has a high specificity for $\mathrm{PCa}$, the presence of GSTP1 hypermethylation in urinary sediments of patients with negative biopsies (33\%) and patients with atypia or highgrade PIN (67\%) suggests that these patients may have occult PCa. ${ }^{11}$

\section{RASSF1A}

RASSF1 has two major transcripts, termed RASSF1A and RASSF1C, which are transcribed from different $\mathrm{CpG}$ island promoters. The promoter of RASSF1A is often hypermethylated in breast, kidney, liver, lung and $\mathrm{PCa}$, whereas the promoter region of RASSF1C is never methylated. RASSF1A reduces tumour growth in vivo and in vitro. ${ }^{12}$ This supports a role for RASSF1A as a tumour suppressor gene. It was shown that RASSF1A binds to microtubules and protects cells from microtubule destabilizing agents. ${ }^{13}$ This interaction contributes to cell cycle regulation and mitotic progression.
Initially no RASSF1A hypermethylation was detected in normal prostate tissue. ${ }^{14,15}$ Recently, methylation of the RASSF1A gene was observed in both premalignant PINs and benign prostatic epithelia. ${ }^{16}$ RASSF1A hypermethylation has been observed in $60 \%-74 \%$ of prostate tumours and in $18.5 \%$ of BPH samples. Furthermore, the methylation frequency is clearly associated with high Gleason score and stage. $^{14,15,17}$ These findings suggest that RASSF1A hypermethylation may distinguish the more aggressive tumours from the indolent ones.

\section{Multiplexed assays}

Multiplexed assays consisting of three or more methylation markers may provide better specificity and sensitivity. Abberant methylation of GSTP1, RASSF1A, retinoic acid receptor $\beta 2$ (RARB) and adenomatous polyposis coli $(A P C)$ in urinary cells, obtained after prostate massage, discriminated malignant from non-malignant cases with $86 \%$ sensitivity and $89 \%$ specificity. ${ }^{18}$ In urine sediments obtained from catherized urine specimens (collected during radical prostatectomy), the combined sensitivity of the methylated genes GSTP1, RASSF1A and $R A R B$ for PCa was $82 \% .{ }^{19}$ The most informative biomarkers for PCa were the hypermethylated promoters of RASSF1A and RARB. The RASSF1A promoter was the most commonly methylated with a frequency of $71 \%$. RARB hypermethylation was more common in men with PCas of high Gleason scores. GSTP1, RARB and RASSF1A are known to accumulate low-level promoter methylation in normal cells of ageing individuals. ${ }^{20}$ Therefore, these genes should be investigated in age-matched negative controls to establish a quantitative cutoff point for the amount of methylation that would indicate the presence of cancer.

Using an assay for GSTP1, RARB and APC on urine samples from patients with serum PSA concentrations $\geqslant 2.5 \mu \mathrm{g} \mathrm{l}^{-1}$, a good correlation of GSTP1 hypermethylation with the number of PCa-positive biopsy cores was observed. ${ }^{21}$ Samples that contained methylation for either GSTP1 or RARB correlated with higher tumour volumes. DNA methylation of the genes GSTP1, RASSF2, HIST1HK4 and TFAP2E had a higher sensitivity for PCa in post-prostate massage 
urine samples compared to plasma samples of the same patients. Furthermore, hypermethylation of the genes RASSF2, HIST1HK4 and TFAP2E was individually as good as GSTP1 in the diagnosis of $\mathrm{PCa}^{22}$

DNA hypermethylation is highly specific for cancer and this epigenetic event is frequent, abundant and stable. Hypermethylated genes have the potential to provide a new generation of cancer urinary biomarkers, although prior to clinical utilisation, these findings require validation in prospective clinical studies.

\section{GENES UNIQUELY EXPRESSED IN PCA}

Microarray studies have been very useful and informative in identifying genes that are consistently up- or downregulated in PCa compared to benign prostate tissue. ${ }^{23}$ These genes can provide PCa-specific biomarkers and provide a greater insight into the aetiology of the disease. For the molecular diagnosis of $\mathrm{PCa}$, genes that are highly upregulated in PCa and have low or normal expression in normal prostate tissue are of special interest. Such genes could enable the detection of one tumour cell in a background of normal cells and thus be applied as a diagnostic marker in PCa detection.

\section{PCA3}

$P C A 3$, formerly known as $D D 3$, was identified using differential display analysis. $P C A 3$ was found to be overexpressed in prostate tumours compared to normal prostate tissue of the same patient using northern blot analysis. ${ }^{24}$ Moreover, PCA3 was found to be strongly overexpressed in more than $95 \%$ of primary PCa specimens and in PCa metastasis. The expression of PCA3 is restricted to prostatic tissue, that is, no expression was found in other normal human tissues. $^{25,26}$ The gene encoding for PCA3 is located on chromosome $9 \mathrm{q} 21.2$. Open reading frame analysis revealed that the PCA3 exons are populated by an unusual number of stop codons. A gene that codes for proteins will typically possess one long open reading frame delimited by a stop codon. The multiplicity of stopcodons across the three reading frames of $P C A 3$ and the lack of an extended open reading frame indicates that $P C A 3$ does not encode a protein and functions as a non-coding RNA. ${ }^{24}$

In the absence of a protein, RT-PCR assays were developed for $P C A 3$. A time-resolved fluorescence-based quantitative RT-PCR assay showed a median 66-fold upregulation of PCA3 in PCa tissue compared to normal prostate tissue. ${ }^{26}$ Moreover, a median upregulation of 11 -fold was found in prostate tissues containing less than $10 \%$ of PCa cells. This indicated that PCA3 was capable of detecting a small number of tumour cells in a background of normal cells. This hypothesis was tested on post-DRE voided urine samples obtained from a group of 108 men who were indicated for prostate biopsies based on a total serum PSA value of $>3 \mathrm{ng} \mathrm{ml}^{-1}$. This test had $67 \%$ sensitivity and $83 \%$ specificity using prostatic biopsies as a gold-standard for the presence of a tumour. Furthermore, this test had a negative predictive value of $90 \%$, which indicates that the quantitative determination of $P C A 3$ transcripts in urinary sediments obtained after DRE bears potential in the reduction of the number of invasive transrectal ultrasoundguided biopsies in this population of men. ${ }^{26}$

The tissue specificity and the high overexpression in prostate tumours indicate that $P C A 3$ is the most $\mathrm{PCa}$-specific gene described so far. PCA3 has been extensively reviewed. ${ }^{27,28}$ Gen-probe Inc. (Hologic Gen-Probe Inc., San Diego, CA, USA) has the exclusive worldwide licence to the PCA3 technology and has successfully transferred the technology to its APTIMA platform. ${ }^{29}$ APTIMA uses transcription-mediated amplification (TMA), which is an RNA transcription amplification system using RNA polymerase and reverse transcriptase to drive the isothermal reaction. This test was released commercially in Europe under the name Progensa PCA3. In several studies using large patient cohorts, $P C A 3$ appears to be superior to serum PSA. ${ }^{30-32}$ A urine PCA3 score of $>35$ had an average sensitivity of $66 \%$ and specificity of $76 \%$ for the prediction of PCa at biopsy, whereas serum PSA had a specificity of only $47 \%$ at about the same sensitivity $(65 \%) .^{33}$ The PCA3 score is not influenced by age, prostatitis, prostate volume and $5 \alpha$-reductase inhibitors. In men with elevated serum PSA levels and one or two previous negative biopsy results, elevated PCA3 scores were correlated to a positive repeat biopsy. Progensa PCA3 was approved by the FDA as a diagnostic test in men with elevated serum PSA and previous negative biopsy result. PCA3 has become the first basis for the molecular diagnostics in clinical urological practice.

Studies on the predictive value of $P C A 3$ for aggressive disease have been contradictory. No significant association between PCA3 score and any prognostic parameter (including stage, Gleason score, tumour volume or extraprostatic extension) was found in three independent studies. ${ }^{34-36}$ Other studies dispute this, and found a correlation between high PCA3 scores and Gleason score $\geqslant 7$ PCa, extracapsular extension and tumour volume. ${ }^{37-39}$ Recently, PCA3 score was found to be a valuable predictor of low-volume, insignificant cancer. ${ }^{38,40}$ As such, PCA3 could be of help in selecting patients for active surveillance. However, in men with significant PCa, PCA3 may not differentiate the agressiveness of a tumour. Thus, a biomarker indicative of tumour aggressiveness is still an unmet need in PCa.

\section{Gene fusions of TMPRSS2 with ETS family members}

Differential gene expression analysis has been successfully used to identify PCa-specific biomarkers by comparing PCa tissues to nonmalignant prostate tissues. Recently, a new biostatistical method called cancer outlier profile analysis (COPA) was used to identify genes that are differentially expressed in a subset of PCas. ${ }^{41}$ COPA identified strong outlier profiles for v-ets erythroblastosis virus E26 oncogene $(E R G)$ and ets variant gene 1 (ETV1) in $57 \%$ of PCa cases. ${ }^{42}$ This was in concordance with the results of a study where PCa-associated ERG overexpression was found in $72 \%$ of PCa cases. ${ }^{43}$ In $>90 \%$ of the cases that overexpressed either ERG or ETV1, a fusion of the 5' untranslated region of the prostate-specific and androgen-regulated transmembrane-serine protease gene (TMPRSS2) with these ETS family members was found. Recently, another fusion between TMPRSS2 and an ETS family member has been described, the TMPRSS2-ETV4 fusion, although this fusion is sporadically found in PCas. ${ }^{44}$ Furthermore, a fusion of TMPRSS2 with ETV5 was found. Overexpression of ETV5 in vitro was shown to induce an invasive transcriptional programme. ${ }^{45}$ These fusions can explain the aberrant androgen-dependent overexpression of ETS family members in subsets of PCa because TMPRSS2 is androgen-regulated. ${ }^{42}$ The discovery of the TMPRSS2-ERG gene fusion and the fact that $E R G$ is the most frequently overexpressed proto-oncogene described in malignant prostate epithelial cells suggests its role in prostate tumourigenesis.

\section{Combination of TMPRSS2-ERG gene fusions with PCA3}

Recently, it was shown that non-invasive detection of TMPRSS2-ERG fusion transcripts is feasible in urinary sediments obtained after DRE using an RT-PCR-based research assay. Due to the high specificity of the test (93\%), the combination of TMPRSS2-ERG fusion transcripts with PCA3 improved the sensitivity from $62 \%$ (PCA3 alone) to $73 \%$ (combined) without compromising the specificity for detecting PCa. ${ }^{46}$ Another study confirmed that PCA3 has the highest sensitivity 
(93\%) and TMPRSS2-ERG fusion transcript has the highest specificity $(87 \%)$ in predicting PCa diagnosis. Combining PCA3, TMPRSS2-ERG and serum PSA in a multivariable algorithm, PCa could be predicted in urine with $80 \%$ sensitivity and $90 \%$ specificity. ${ }^{47}$ In another study, no TMPRSS2-ERG fusion transcripts were found in urine samples obtained from females, healthy young men and post-radical prostatectomy patients. ${ }^{48}$ In $34.8 \%$ of the urine samples from PCa patients, TMPRSS2-ERG fusion transcripts were found compared to $18.2 \%$ of the urine specimen from men with negative biopsies. Although this study is limited in sample size, it confirms that this marker can be used as a diagnostic tool for PCa.

Using real-time PCR, a positive correlation was found between TMPRSS2-ERG fusion transcripts in urine collected after prostate massage and a high serum PSA, pathological stage and Gleason score. ${ }^{49}$ TMPRSS2-ERG gene fusions were highly associated with aggressive Gleason score $\geqslant 7$ and PCa-related death. ${ }^{50}$ Elevated urine TMPRSS2-ERG was also associated with the presence of PCa and Epstein criteria for significant PCa (Gleason score, tumour volume, $\%$ of cancer per biopsy core and number of positive cores).

It was shown that men with extremes of TMPRSS2-ERG+PCA3 have different risks of cancer and aggressiveness of the cancer on biopsy. In combination with other clinical pathological parameters (combined in the multivariate prostate cancer prevention trial risk calculator), urine TMPRSS2-ERG+PCA3 may guide the urgency of biopsy after the detection of an elevated serum PSA. ${ }^{51}$

Serine peptidase inhibitor Kazal type 1 (SPINK1), golgi phosphoprotein 2 (GOLPH2) and TMPRSS2-ERG were, like PCA3, independent predictors of $\mathrm{PCa}$ upon repeat biopsy. ${ }^{52}$ By combining $P C A 3$ with these markers in a quantitative multiplexed RT-PCR analysis, the ROC AUC value improved from 0.66 ( $P C A 3$ alone) to 0.76 . This multiplexed urine-based assay had $66 \%$ sensitivity and $76 \%$ specificity for detecting PCa in repeat biopsies. In men with elevated serum PSA, urine TMPRSS2-ERG in combination with PCA3 may aid in decisionmaking on biopsy and management of the disease. By combining SPINK1 with TMPRSS2-ERG and PCA3 in a multiplexed panel, the risk stratification of PCa may be further improved.

\section{a-methylacyl-CoA racemase (AMACR)}

The gene coding for AMACR on chromosome $5 \mathrm{p} 13$ was found to be consistently upregulated in PCa. This enzyme plays a critical role in peroxisomal $\beta$-oxidation of branched chain fatty acid molecules obtained from dairy and beef. ${ }^{53}$ Interestingly, the consumption of dairy and beef has been associated with an increased risk of $\mathrm{PCa}{ }^{54}$

In clinical PCa tissue, a ninefold overexpression of AMACR mRNA was found compared to normal prostate tissue. Immunohistochemical studies and western blot analyses have confirmed the upregulation of AMACR at the protein level. Furthermore, it was shown that $88 \%$ of PCa cases and both untreated metastases and castration resistant PCas were strongly positive for AMACR. ${ }^{55}$ AMACR expression was not detected in atrophic glands, basal cell hyperplasia and urothelial epithelium or metaplasia. Immunohistochemical studies also showed that AMACR expression in needle biopsies had a $97 \%$ sensitivity and a $100 \%$ specificity for PCa detection. ${ }^{56}$ Combined with a staining for p63, a basal cell marker absent in PCa, AMACR greatly facilitated the identification of malignant prostate cells. ${ }^{57,58}$ Currently, the accuracy and specificity of AMACR in the detection of PCa in biopsy specimens is regarded as an improvement over the serum PSA test. $^{59}$ Its high expression and cancer cell specificity implies that AMACR may also be a candidate for the development of molecular probes that may facilitate the identification of PCa using non-invasive imaging modalities. ${ }^{55}$

There have been many efforts to develop a body fluid-based assay for AMACR. First, an AMACR activity assay used on extracts derived from prostate needle biopsy specimen had $92.3 \%$ sensitivity and 89.2\% specificity for PCa detection. ${ }^{60}$ Second, an AMACR immune reactivity assay showed $71.8 \%$ specificity and $61.6 \%$ sensitivity in distinguishing the sera of PCa patients from those of healthy controls, and could be used in combination with serum PSA to reduce the number of unnecessary biopsies. ${ }^{61}$ Third, AMACR protein western blot analysis on urine samples obtained after prostate massage had a sensitivity of $100 \%$, a specificity of $58 \%$, a positive predictive value of $72 \%$ and a negative predictive value of $88 \%$ for PCa. ${ }^{62}$ Fourth, a small study indicated that $A M A C R$ mRNA-based quantitative real-time PCR analysis on urine samples obtained after prostate massage has the potential to exclude the patients with clinically insignificant disease when AMACR expression is normalized for PSA. ${ }^{63}$ Fifth, when AMACR transcripts were measured and combined with PCA3 scores in post-DRE urinary sediments of 43 men with and 49 men without PCa, this duplex test had $81 \%$ sensitivity and $84 \%$ specificity for the detection of PCa. ${ }^{64}$ Therefore, AMACR-based assays for the detection of PCa in urine specimen are promising, although additional investigation is needed to validate the clinical usefulness of this urinary biomarker.

\section{Prostate-specific membrane antigen (PSMA)}

PSMA is a transmembrane glycoprotein that is expressed on the surface of prostate epithelial cells. It was shown that PSMA is upregulated in PCa tissue compared to benign prostate tissues. No overlap in PSMA expression was found between $\mathrm{BPH}$ and $\mathrm{PCa}$, indicating that PSMA is a very promising diagnostic marker. ${ }^{65}$ Recently, it was shown that high PSMA expression in PCa cases correlated with tumour grade, pathological stage, aneuploidy and biochemical recurrence. Furthermore, increased PSMA mRNA expression in primary PCas and metastasis correlated with PSMA protein overexpression. ${ }^{66}$ Its clinical utility as a diagnostic or prognostic marker for PCa has been hindered by the lack of a sensitive immunoassay for this protein. However, a combination of ProteinChip (Ciphergen Biosystems, Fremont, CA, USA) arrays and surface-enhanced laser desorption ionisation/time-of-flight (SELDI-TOF) mass spectrometry has led to the introduction of a protein biochip immunoassay for the quantification of serum PSMA. It was shown that the average serum PSMA levels for PCa patients were significantly higher compared to those of men with BPH and healthy controls. ${ }^{67}$ These findings implicate a role for serum PSMA to distinguish men with $\mathrm{BPH}$ from PCa patients. However, further studies are needed to assess its diagnostic value.

RT-PCR studies have shown that PSMA in combination with its splice variant $P S M^{\prime}$ could be used as a prognostic marker for PCa. In the normal prostate, $P S M^{\prime}$ expression is higher than PSMA expression, whereas in PCa tissues, the PSMA expression is more dominant. Therefore, the ratio of PSMA to $P S M^{\prime}$ is highly indicative for disease progression. Designing a quantitative PCR analysis which discriminates between the two PSMA forms could yield another application for PSMA in diagnosis and prognosis of $\mathrm{PCa}{ }^{65,68}$

In men with serum PSA values between 4 and $10 \mathrm{ng} \mathrm{ml}^{-1}$ with no previous biopsies, PSMA mRNA measured in post-prostate massage urinary sediments was superior to $P C A 3$ and $P S G R$ in predicting cancer upon biopsy. At $70 \%$ specificity, the sensitivity for PSMA, PCA3 and $P S G R$ was $64 \%, 46 \%$ and $61 \%$, respectively. A combination of the three markers resulted in $68 \%$ sensitivity at $70 \%$ specificity. ${ }^{69}$ 
Because of its specific expression on prostate epithelial cells and its upregulation in PCa, PSMA has also become the target for therapies. The proposed strategies range from targeted toxins and radio nuclides to immunotherapeutic agents. First-generation products have entered clinical testing. ${ }^{70}$

\section{MICRORNAS (MIRNAS)}

Mature miRNAs are non-coding RNAs that play key roles in the regulation of gene expression. They are short, single-stranded RNA molecules of $\sim 22$ nucleotides in length that bind to complementary sequences in the $3^{\prime}$ UTR of multiple target mRNAs, usually resulting in their silencing. miRNAs have shown to be involved in a wide range of biological processes such as cell cycle control, apoptosis and several developmental and physiological processes. miRNAs have also been implicated in a number of diseases including a broad range of cancers, heart disease and neurological diseases. Their small size and the fact that they are secreted within microvesicles protect the miRNAs from RNase. $^{71,72}$ Consequently, the detection of miRNAs in body fluids represents a promising non-invasive diagnostic utility for $\mathrm{PCa}$. miRNAs are mostly studied in serum and plasma. Independent studies have shown that there are two promising miRNAs in the blood circulation, miR-141 and miR-375. In patients with metastatic PCa plasma miR-141 levels were 46-fold higher compared to the healthy individuals. ${ }^{73,74}$ Elevated plasma levels of miR-141 and miR-375 could discriminate men with metastatic disease from men without metastases. ${ }^{75}$ Elevated serum levels of these two miRNAs were associated with higher Gleason score and positive lymph node status. ${ }^{76}$ In microvesicle and exosome portions taken from sera from men with metastatic disease, both miRNAs were significantly increased in both portions compared to those obtained from men with non-recurring disease. $^{75}$

Since changes in miRNA levels occur in serum and plasma of PCa patients, there may be a possibility that some of these changes may also occur in urine. Until now, only one study for miRNA in urine was reported. ${ }^{75}$ Five of the miRNAs that were differentially quantified in PCa patients compared to controls (miR-107, miR-574-3p, miR375, miR200b and miR-141) were successfully quantified in urine of men with cancer. Although miR-141 and miR-375 were useful serum and plasma biomarkers for metastatic disease, they were unable to discriminate men with PCa compared to healthy controls in urine. However, miRNAs miR-107 and miR-574-3p were significantly higher in the urine of men with PCa when compared to controls. They appeared to be even better than PCA3 normalized to PSA in identifying the presence of PCa in urine samples.

Several miRNA biomarkers have been described to be associated with PCa onset or progression. However, the analysis on circulating miRNAs for PCa is still in its infancy. The analysis of miRNAs in body fluids is a challenge with respect to the extraction of RNA from limited sources of biomaterial. Although many of the miRNA studies as potential biomarkers for PCa were done on serum and plasma specimen, the single study in urine holds promise that they can be used for blood-based as well as urine-based assays. The promising results from these initial studies need to be validated in larger patient cohorts with good controls (e.g., healthy males, men with BPH and/or prostatitis) and comprehensive follow-up data using robust methods and standards for miRNA extraction and detection. ${ }^{77}$

\section{EXOSOMES}

Exosomes are small tissue-derived vesicles with a diameter of 50-150 $\mathrm{nm}$ that are shed by many mammalian cell types, including malignant cells. The protein and RNA content of exosomes represent their tissue origin and associate with the original cellular conditions. ${ }^{78}$ Exosomes are enriched in unique mRNA transcripts specific to tumour cells compared to the tissue donor cells. This can be explained by the fact that the exosomes contain less ribosomal RNA, thereby increasing the relative amount of mRNA. ${ }^{79}$ Exosomes are present in different body fluids (e.g., urine, semen, blood and malignant ascites). The celltype specificity of these exosomes makes them an attractive source of new tissue or disease-specific biomarker targets such as proteins, mRNAs and miRNAs. ${ }^{80-83}$ For PCa, the urinary exosomes hold promise as a non-invasive source of biomarkers.

Recently, it was shown that it is technically feasible to study urinary exosomes of PCa patients for evaluation of the clinical status of disease, although the current extraction protocols have been labourintensive and not optimal. ${ }^{79,84,85} \mathrm{PCa}$ patients had higher levels of urinary exosomes than the healthy donors. Prostate markers (e.g., PSA and PSMA) were not expressed in specimen from healthy donors, suggesting that few exosomes in healthy donor urines are of prostatic origin. ${ }^{85}$ The membranes of exosomes are resistant to the osmeolytic and proteolytic activity of urine. This indicates that exosomes are quite stable in this body fluid.

PSA, TMPRSS2-ERG gene fusion and PCA3 mRNA transcripts can be detected in the urinary exosomal fraction after a mild prostate massage. ${ }^{85}$ Prostate massage is necessary to increase the exosomal secretion into the urethra and subsequently into the collected urine fraction which is in concordance with earlier published findings of shedding prostate tumour cells in the urine. ${ }^{26,33,52}$

After a mild prostate massage, TMPRSS2-ERG gene fusions were detected in 2/4 patients with high Gleason scores and in none of the patients with low Gleason scores. PCA3 transcripts were detected in all of the patients. None of the patients under androgen deprivation therapy (ADT) had detectable levels of PSA, TMPRSS2-ERG gene fusion or PCA3 mRNA transcripts. This loss of expression of these androgen-regulated biomarkers correlated with tumour regression and a positive response to the ADT. ${ }^{85}$ Independently, others showed that there is a decrease in the amount of urinary exosomes under ADT. ${ }^{84}$

Although these results are promising, more studies are necessary to assess the clinical value of urinary exosomes. Modifications in collection, storage and handling of urine samples can improve the isolation of the urinary exosomes providing a simple enrichment of potential disease biomarkers.

\section{CONCLUSION}

There is an urgent need to discover more accurate non-invasive tests for PCa diagnosis and to allow the stratification of patients with lifethreatening PCa. Because of the ease of collection, and the fact that prostate cells are directly released into the urethra through prostatic ducts after DRE or prostate massage, urine has become the future of non-invasive biomarker testing. Many studies have shown the feasibility of urine for the non-invasive detection of PCa. Recently, it was shown that RNA biomarkers can also be detected in urinary exosomes, making them promising for biomarkers research as well.

Biomarker research is in focus at many laboratories and several biomarkers are promising due to their specificity for the disease in tissue. To date, only few of these biomarkers have shown to be useful as urinary marker. Two PCa-specific RNA-based biomarkers have been identified (PCA3 and TMPRSS2-ERG gene fusions). The recent FDA approval of $P C A 3$ has led to its introduction in clinical practice and the combination of both markers has been marketed for clinical use as 
well. Compared to single biomarkers, the combination of several biomarkers considerably improves the prediction of PCa in urine samples which is consistent with the heterogeneity of the disease.

In the era of individualized therapy, the biomarker combinations are necessary to not only predict PCa at biopsy, but also the aggressiveness of the cancer. Preliminary results show that the PCa-specific TMPRSS2-ERG gene fusion may be indicative of aggressiveness of cancer upon biopsy, although further studies are warranted. In PCa biomarker development, the greatest unmet need remains: a biomarker that stratifies men at risk of aggressive PCa, eventually leading to a reduction of unnecessary interventions.

\section{COMPETING FINANCIAL INTERESTS}

All authors declare that there are no competing financial interests.

1 Ferlay J, Parkin DM, Steliarova-Foucher E. Estimates of cancer incidence and mortality in Europe in 2008. Eur J Cancer 2010; 46: 765-81.

2 Siegel R, Naishadham D, Jemal A. Cancer statistics, 2012. CA Cancer J Clin 2012; 62: 10-29.

3 Roddam AW, Duffy MJ, Hamdy FC, Ward AM, Patnick J et al. Use of prostate-specific antigen (PSA) isoforms for the detection of prostate cancer in men with a PSA level of 2-10 ng/ml: systematic review and meta-analysis. Eur Urol 2005; 48: 386-99; discussion 98-9.

4 Li S, Hursting SD, Davis BJ, McLachlan JA, Barrett JC. Environmental exposure, DNA methylation, and gene regulation: lessons from diethylstilbesterol-induced cancers. Ann N Y Acad Sci 2003; 983: 161-9.

5 Jones PA, Baylin SB. The fundamental role of epigenetic events in cancer. Nat Rev Genet 2002; 3: 415-28.

6 Goessl C, Krause H, Muller M, Heicappell R, Schrader M et al. Fluorescent methylation-specific polymerase chain reaction for DNA-based detection of prostate cancer in bodily fluids. Cancer Res 2000; 60: 5941-5.

7 Nakayama M, Bennett CJ, Hicks JL, Epstein JI, Platz EA et al. Hypermethylation of the human glutathione $S$-transferase-pi gene (GSTP1) $\mathrm{CpG}$ island is present in a subset of proliferative inflammatory atrophy lesions but not in normal or hyperplastic epithelium of the prostate: a detailed study using laser-capture microdissection. Am J Pathol 2003; 163: 923-33.

8 Lee WH, Morton RA, Epstein JI, Brooks JD, Campbell PA et al. Cytidine methylation of regulatory sequences near the pi-class glutathione S-transferase gene accompanies human prostatic carcinogenesis. Proc Natl Acad Sci U S A 1994; 91: 11733-7.

9 Goessl C, Muller M, Heicappell R, Krause H, Straub B et al. DNA-based detection of prostate cancer in urine after prostatic massage. Urology 2001; 58: 335-8.

10 Woodson K, O'Reilly KJ, Hanson JC, Nelson D, Walk EL et al. The usefulness of the detection of GSTP1 methylation in urine as a biomarker in the diagnosis of prostate cancer. J Urol 2008; 179: 508-11; discussion 11-2.

11 Gonzalgo ML, Pavlovich CP, Lee SM, Nelson WG. Prostate cancer detection by GSTP1 methylation analysis of postbiopsy urine specimens. Clin Cancer Res 2003; 9: 26737.

12 Dammann R, Schagdarsurengin U, Strunnikova M, Rastetter M, Seidel C et al. Epigenetic inactivation of the Ras-association domain family 1 (RASSF1A) gene and its function in human carcinogenesis. Histol Histopathol 2003; 18: 665-77.

13 Gordon M, EI-Kalla M, Baksh S. RASSF1 polymorphisms in cancer. Mol Biol Int 2012; 2012: 365213 .

14 Liu L, Yoon JH, Dammann R, Pfeifer GP. Frequent hypermethylation of the RASSF1A gene in prostate cancer. Oncogene 2002; 21: 6835-40.

15 Kuzmin I, Gillespie JW, Protopopov A, Geil L, Dreijerink K et al. The RASSF1A tumor suppressor gene is inactivated in prostate tumors and suppresses growth of prostate carcinoma cells. Cancer Res 2002; 62: 3498-502.

16 Aitchison A, Warren A, Neal D, Rabbitts P. RASSF1A promoter methylation is frequently detected in both pre-malignant and non-malignant microdissected prostatic epithelial tissues. Prostate 2007; 67: 638-44.

17 Kawamoto K, Okino ST, Place RF, Urakami S, Hirata H et al. Epigenetic modifications of RASSF1A gene through chromatin remodeling in prostate cancer. Clin Cancer Res 2007; 13: 2541-8.

18 Roupret M, Hupertan V, Yates DR, Catto JW, Rehman I et al. Molecular detection of localized prostate cancer using quantitative methylation-specific PCR on urinary cells obtained following prostate massage. Clin Cancer Res 2007; 13: 1720-5.

19 Daniunaite K, Berezniakovas A, Jankevicius F, Laurinavicius A, Lazutka JR et al. Frequent methylation of RASSF1 and RARB in urine sediments from patients with early stage prostate cancer. Medicina (Kaunas) 2011; 47: 147-53.

20 Kwabi-Addo B, Chung W, Shen L, Ittmann M, Wheeler T et al. Age-related DNA methylation changes in normal human prostate tissues. Clin Cancer Res 2007; 13: 3796-802.

21 Vener T, Derecho C, Baden J, Wang H, Rajpurohit Y et al. Development of a multiplexed urine assay for prostate cancer diagnosis. Clin Chem 2008; 54: 874-82.
22 Payne SR, Serth J, Schostak M, Kamradt J, Strauss A et al. DNA methylation biomarkers of prostate cancer: confirmation of candidates and evidence urine is the most sensitive body fluid for non-invasive detection. Prostate 2009; 69: 1257-69.

23 Kumar-Sinha C, Rhodes DR, Yu J, Chinnaiyan AM. Prostate cancer biomarkers: a current perspective. Expert Rev Mol Diagn 2003; 3: 459-70.

24 Bussemakers MJ, van Bokhoven A, Verhaegh GW, Smit FP, Karthaus HF et al. DD3: a new prostate-specific gene, highly overexpressed in prostate cancer. Cancer Res 1999; 59: 5975-9.

25 de Kok JB, Verhaegh GW, Roelofs RW, Hessels D, Kiemeney LA et al. DD3(PCA3), a very sensitive and specific marker to detect prostate tumors. Cancer Res 2002; 62: 2695-8.

26 Hessels D, Klein Gunnewiek JM, van Oort I, Karthaus HF, van Leenders GJ et al. DD3(PCA3)-based molecular urine analysis for the diagnosis of prostate cancer. Eur Urol 2003; 44: 8-15; discussion -6.

27 Hessels D, Schalken JA. The use of PCA3 in the diagnosis of prostate cancer. Nat Rev Urol 2009; 6: 255-61.

28 Vlaeminck-Guillem V, Ruffion A, Andre J, Devonec M, Paparel P. Urinary prostate cancer 3 test: toward the age of reason? Urology 2010; 75: 447-53.

29 Groskopf J, Aubin SM, Deras IL, Blase A, Bodrug S et al. APTIMA PCA3 molecular urine test: development of a method to aid in the diagnosis of prostate cancer. Clin Chem 2006; 52: 1089-95.

30 Deras IL, Aubin SM, Blase A, Day JR, Koo S et al. PCA3: a molecular urine assay for predicting prostate biopsy outcome. J Urol 2008; 179: 1587-92.

31 Chun FK, de la Taille A, van Poppel H, Marberger M, Stenzl A et al. Prostate cancer gene 3 (PCA3): development and internal validation of a novel biopsy nomogram. Eur Urol 2009; 56: 659-67.

32 Tosoian JJ, Loeb S, Kettermann A, Landis P, Elliot DJ et al. Accuracy of PCA3 measurement in predicting short-term biopsy progression in an active surveillance program. J Urol 2010; 183: 534-8.

33 van Gils MP, Hessels D, van Hooij O, Jannink SA, Peelen WP et al. The time-resolved fluorescence-based PCA3 test on urinary sediments after digital rectal examination; a dutch multicenter validation of the diagnostic performance. Clin Cancer Res 2007; 13: 939-43.

34 Hessels D, van Gils MP, van Hooij O, Jannink SA, Witjes JA et al. Predictive value of PCA3 in urinary sediments in determining clinico-pathological characteristics of prostate cancer. Prostate 2010; 70: 10-6.

35 van Gils MP, Hessels D, Hulsbergen-van de Kaa CA, Witjes JA, Jansen CF et al. Detailed analysis of histopathological parameters in radical prostatectomy specimens and PCA3 urine test results. Prostate 2008; 68: 1215-22.

36 Liss MA, Santos R, Osann K, Lau A, Ahlering TE et al. PCA3 molecular urine assay for prostate cancer: association with pathologic features and impact of collection protocols. World J Urol 2011; 29: 683-8.

37 Marks LS, Fradet Y, Deras IL, Blase A, Mathis J et al. PCA3 molecular urine assay for prostate cancer in men undergoing repeat biopsy. Urology 2007; 69: 532-5.

38 Nakanishi H, Groskopf J, Fritsche HA, Bhadkamkar V, Blase A et al. PCA3 molecular urine assay correlates with prostate cancer tumor volume: implication in selecting candidates for active surveillance. J Urol 2008; 179: 1804-9; discussion 9-10.

39 Whitman EJ, Groskopf J, Ali A, Chen Y, Blase A et al. PCA3 score before radical prostatectomy predicts extracapsular extension and tumor volume. J Urol 2008; 180: 1975-8; discussion 8-9.

40 Auprich M, Chun FK, Ward JF, Pummer K, Babaian R et al. Critical assessment of preoperative urinary prostate cancer antigen 3 on the accuracy of prostate cancer staging. Eur Urol 2011; 59: 96-105.

41 Kumar-Sinha C, Tomlins SA, Chinnaiyan AM. Evidence of recurrent gene fusions in common epithelial tumors. Trends Mol Med 2006; 12: 529-36.

42 Tomlins SA, Rhodes DR, Perner S, Dhanasekaran SM, Mehra R et al. Recurrent fusion of TMPRSS2 and ETS transcription factor genes in prostate cancer. Science 2005; 310: 644-8.

43 Petrovics G, Liu A, Shaheduzzaman S, Furusato B, Sun C et al. Frequent overexpression of ETS-related gene-1 (ERG1) in prostate cancer transcriptome. Oncogene 2005; 24: 3847-52.

44 Tomlins SA, Mehra R, Rhodes DR, Smith LR, Roulston D et al. TMPRSS2:ETV4 gene fusions define a third molecular subtype of prostate cancer. Cancer Res 2006; 66: 3396-400.

45 Helgeson BE, Tomlins SA, Shah N, Laxman B, Cao Q et al. Characterization of TMPRSS2:ETV5 and SLC45A3:ETV5 gene fusions in prostate cancer. Cancer Res 2008; 68: 73-80.

46 Hessels D, Smit FP, Verhaegh GW, Witjes JA, Cornel EB et al. Detection of TMPRSS2ERG fusion transcripts and prostate cancer antigen 3 in urinary sediments may improve diagnosis of prostate cancer. Clin Cancer Res 2007; 13: 5103-8.

47 Salami SS, Schmidt F, Laxman B, Regan MM, Rickman DS et al. Combining urinary detection of TMPRSS2:ERG and PCA3 with serum PSA to predict diagnosis of prostate cancer. Urol Oncol; e-pub ahead of print 19 May 2011; doi:10.1016/ j.urolonc.2011.04.001.

48 Nguyen PN, Violette P, Chan S, Tanguay S, Kassouf W et al. A panel of TMPRSS2:ERG fusion transcript markers for urine-based prostate cancer detection with high specificity and sensitivity. Eur Urol 2011; 59: 407-14.

49 Rostad K, Hellwinkel OJ, Haukaas SA, Halvorsen OJ, Oyan AM et al. TMPRSS2:ERG fusion transcripts in urine from prostate cancer patients correlate with a less favorable prognosis. APMIS 2009; 117: 575-82.

50 Demichelis F, Fall K, Perner S, Andren O, Schmidt F et al. TMPRSS2:ERG gene fusion associated with lethal prostate cancer in a watchful waiting cohort. Oncogene 2007; 26: 4596-9. 
51 Tomlins SA, Aubin SM, Siddiqui J, Lonigro RJ, Sefton-Miller L et al. Urine TMPRSS2:ERG fusion transcript stratifies prostate cancer risk in men with elevated serum PSA. Sci Trans/ Med 2011; 3: 94 ra72.

52 Laxman B, Morris DS, Yu J, Siddiqui J, Cao J et al. A first-generation multiplex biomarker analysis of urine for the early detection of prostate cancer. Cancer Res 2008; 68: 645-9.

53 Schmitz W, Albers C, Fingerhut R, Conzelmann E. Purification and characterization of an alpha-methylacyl-coa racemase from human liver. Eur J Biochem 1995; 231: 815-22.

54 Michaud DS, Augustsson K, Rimm EB, Stampfer MJ, Willet WC et al. A prospective study on intake of animal products and risk of prostate cancer. Cancer Causes Control 2001; 12: 557-67.

55 Luo J, Zha S, Gage WR, Dunn TA, Hicks JL et al. Alpha-methylacyl-coa racemase: new molecular marker for prostate cancer. Cancer Res 2002; 62: 2220-6.

56 Rubin MA, Zhou M, Dhanasekaran SM, Varambally S, Barrette TR et al. Alphamethylacyl coenzyme a racemase as a tissue biomarker for prostate cancer. JAMA 2002; 287: 1662-70.

57 Fleshman RL, MacLennan GT. Immunohistochemical markers in the diagnosis of prostate cancer. J Urol 2005; 173: 1759.

58 Jiang Z, Li C, Fischer A, Dresser K, Woda BA. Using an amacr (p504s)/34betae12/p63 cocktail for the detection of small focal prostate carcinoma in needle biopsy specimens. Am J Clin Pathol 2005; 123: 231-6.

59 Jiang Z, Woda BA, Wu CL, Yang XJ. Discovery and clinical application of a nove prostate cancer marker: alpha-methylacyl coa racemase (p504s). Am J Clin Patho 2004; 122: 275-89.

60 Kumar-Sinha C, Shah RB, Laxman B, Tomlins SA, Harwood J et al. Elevated alphamethylacyl-coa racemase enzymatic activity in prostate cancer. Am J Pathol 2004; 164: 787-93.

61 Sreekumar A, Laxman B, Rhodes DR, Bhagavathula S, Harwood J et al. Humora immune response to alpha-methylacyl-coa racemase and prostate cancer. $J$ Nat Cancer Inst 2004; 96: 834-43.

62 Rogers CG, Yan G, Zha S, Gonzalgo ML, Isaacs WB et al. Prostate cancer detection on urinalysis for alpha methylacyl coenzyme a racemase protein. J Urol 2004; 172 1501-3.

63 Zielie PJ, Mobley JA, Ebb RG, Jiang Z, Blute RD et al. A novel diagnostic test for prostate cancer emerges from the determination of alpha-methylacyl-coenzyme a racemase in prostatic secretions. J Urol 2004; 172: 1130-3.

64 Ouyang B, Bracken B, Burke B, Chung E, Liang J et al. A duplex quantitative polymerase chain reaction assay based on quantification of alpha-methylacyl-coa racemase transcripts and prostate cancer antigen 3 in urine sediments improved diagnostic accuracy for prostate cancer. J Urol 2009; 181: 2508-13; discussion $13-4$.

65 Burger MJ, Tebay MA, Keith PA, Samaratunga HM, Clements J et al. Expression analysis of delta-catenin and prostate-specific membrane antigen: their potential as diagnostic markers for prostate cancer. Int J Cancer 2002; 100: 228-37.

66 Ross JS, Sheehan CE, Fisher HA, Kaufman RP Jr, Kaur P et al. Correlation of primary tumor prostate-specific membrane antigen expression with disease recurrence in prostate cancer. Clin Cancer Res 2003; 9: 6357-62.
67 Xiao Z, Adam BL, Cazares LH, Clements MA, Davis JW et al. Quantitation of serum prostate-specific membrane antigen by a novel protein biochip immunoassay discriminates benign from malignant prostate disease. Cancer Res 2001; 61 6029-33.

68 Zhang L, Wang CY, Yang R, Shi J, Fu R et al. Real-time quantitative RT-PCR assay of prostate-specific antigen and prostate-specific membrane antigen in peripheral blood for detection of prostate cancer micrometastasis. Urol Oncol 2008; 26: 634-40.

69 Rigau M, Ortega I, Mir MC, Ballesteros C, Garcia M et al. A three-gene panel on urine increases PSA specificity in the detection of prostate cancer. Prostate $2011 ; 71$ 1736-45.

70 Elsasser-Beile $\mathrm{U}$, Buhler $\mathrm{P}$, Wolf $\mathrm{P}$. Targeted therapies for prostate cancer against the prostate specific membrane antigen. Curr Drug Targets 2009; 10: 118-25.

71 Valadi H, Ekstrom K, Bossios A, Sjostrand M, Lee JJ et al. Exosome-mediated transfer of mrnas and micrornas is a novel mechanism of genetic exchange between cells. Nat Cell Biol 2007: 9: 654-9.

72 Wang K, Zhang S, Weber J, Baxter D, Galas DJ. Export of micrornas and micrornaprotective protein by mammalian cells. Nucleic Acids Res 2010; 38: 7248-59.

73 Mitchell PS, Parkin RK, Kroh EM, Fritz BR, Wyman SK et al. Circulating micrornas as stable blood-based markers for cancer detection. Proc Natl Acad Sci U S A2008; 105 10513-8.

74 Yaman Agaoglu F, Kovancilar M, Dizdar Y, Darendeliler E, Holdenrieder S et al Investigation of mir-21, mir-141, and mir-221 in blood circulation of patients with prostate cancer. Tumour Biol 2011; 32: 583-8.

75 Bryant RJ, Pawlowski T, Catto JW, Marsden G, Vessella RL et al. Changes in circulating microrna levels associated with prostate cancer. Br J Cancer 2012; 106: 768-74.

76 Brase JC, Wuttig D, Kuner R, Sultmann H. Serum micrornas as non-invasive biomarkers for cancer. Mol Cancer 2010; 9: 306.

77 Kuner R, Brase JC, Sultmann H, Wuttig D. Microrna biomarkers in body fluids of prostate cancer patients. Methods 2013; 59: 132-7.

78 Gonzalez-Begne M, Lu B, Han X, Hagen FK, Hand AR et al. Proteomic analysis of human parotid gland exosomes by multidimensional protein identification technology (MUDPIT). J Proteome Res 2009; 8: 1304-14.

79 Skog J, Wurdinger T, van Rijn S, Meijer DH, Gainche L et al. Glioblastoma microvesicles transport RNA and proteins that promote tumour growth and provide diagnostic biomarkers. Nat Cell Biol 2008; 10: 1470-6.

80 Mathivanan S, Simpson RJ. Exocarta: a compendium of exosomal proteins and rna. Proteomics 2009; 9: 4997-5000.

81 Rabinowits G, Gercel-Taylor C, Day JM, Taylor DD, Kloecker GH. Exosomal microrna: diagnostic marker for lung cancer. Clin Lung Cancer 2009; 10: 42-6.

82 Taylor DD, Gercel-Taylor C. Microrna signatures of tumor-derived exosomes as diagnostic biomarkers of ovarian cancer. Gynecol Oncol 2008; 110: 13-21.

83 Hunter MP, Ismail N, Zhang X, Aguda BD, Lee EJ et al. Detection of microrna expression in human peripheral blood microvesicles. PLoS One 2008; 3: e3694.

84 Mitchell PJ, Welton J, Staffurth J, Court J, Mason MD et al. Can urinary exosomes act as treatment response markers in prostate cancer? J Trans/ Med 2009; 7: 4.

85 Nilsson J, Skog J, Nordstrand A, Baranov V, Mincheva-Nilsson L et al. Prostate cancerderived urine exosomes: a novel approach to biomarkers for prostate cancer. $\mathrm{Br} J$ Cancer 2009; 100: 1603-7. 